
\title{
$\angle S$ Research Square \\ Melatonin supplementation and outcomes after assisted reproductive technology: A systematic review and meta-analyses
}

\section{Jing Zhao}

Xiangya Hospital Central South University

Jie Hao

Xiangya Hospital Central South University

\section{Bin Xu}

Xiangya Hospital Central South University

\section{Yonggang Wang}

Xiangya Hospital Central South University

Yanping Li ( $\sim$ LYP7798@126.COM )

Xiangya Hospital Central South University https://orcid.org/0000-0002-3658-3274

\section{Research}

Keywords: Melatonin, assisted reproductive technology (ART), in vitro fertilization (IVF), clinical pregnancy

Posted Date: May 10th, 2021

DOI: https://doi.org/10.21203/rs.3.rs-88552/v2

License: (1) This work is licensed under a Creative Commons Attribution 4.0 International License. Read Full License 


\section{Abstract}

To assess the effect of melatonin (MT) supplementation on the outcomes of ART. A meta-analysis and systematic review was conducted. Eleven studies were included in this meta-analysis. Clinical pregnancy rate (CPR), live birth rate (LBR), Miscarriage rate (MR), fertilization rate (FR), Number of oocyte, Mll oocyte, top-quality embryo were reported in $10,3,6,7,9,8$, and 6 studies, respectively. MT supplementation significantly increased the CPR, the No. of MIl oocyte, the No. of top-quality embryo, and the FR. However, there was no significant difference in LBR, No. of oocyte, and the MR. When studies were sub-grouped by the interventions, no matter the control group is Ml+FA or placebo/none, MT supplementation increased No. of MII oocyte and No. of top embryo, whereas showed similar CPR. When studies were sub-grouped according to women's characteristic, MT supplementation showed no significant benefit on CPR in women with PCOS, with normal ovary function, and with previous low fertilization or poor-quality embryo. However, MT supplementation increased the No of MII in women with PCOS, but did not show benefit in women with normal ovary function. MT supplementation may not improve the CPR and LBR of ART. But MT seems be beneficial to the quality of oocyte and embryo, especially for women with PCOS and DOR. Further well-designed studies are needed before the recommendation of its clinical use.

\section{Introduction}

Assisted reproductive technology (ART), which was a very effective and widely used treatment for infertility, has been made great progress since the first tube baby born in 1978 (1). However, according to the present reports, the successfully clinical pregnancy rate (CPR) and the live birth rate (LBR) have been hovering at approximately $50 \%$ and $30 \%$, respectively, with a $51 \%$ of cumulative live birthrates (CLBR) within 3 treatment cycles (2). How to enhance the success of ART has been the focus of research in this field.

In the process of ART, the failure in fertilization, embryo development, and implantation may result from poor oocyte quality (3). While the underlying mechanism leading to poor oocyte quality has not been fully elucidated, oxidative stress is considered to be a possible reason for poor oocyte quality, which may impair female reproductive ability (4). Limiting oxidative stress in oocyte resulted from ROS can produce top-quality embryo. Therefore, it is proposed to use anti-oxidants to mitigate the harmful effects of excessive ROS and improve success rate.

MT, a hormone primarily secreted by the pineal gland. MT and its metabolites can directly mitigate neutralize ROS in human tissues, and can up regulate the gene expression of various antioxidant enzymes, and reduce oxidative damage to cells (5).

Therefore, Many clinics have introduced MT supplementation into their routine practice, and investigated the effect of this intervention on reproductive outcomes. Some studies found improved oocyte quality, embryo quality, and enhanced outcome of ART (6-8). One the contrary, some studies failed to show any beneficial effect with MT supplementation in infertile women undergoing ART(9). So far, there were two systematic reviews with inconsistent results. One meta-analysis included 5 studies and failed to show beneficial effect of MT supplementation on the most important reproductive outcomes (10). The other concluded that supplementation of MT significantly increased the CPR (11). 
Therefore, it was necessary to evaluated weather supplementation of MT have beneficial effect on outcome of ART. This systematic review and meta-analysis aim to clarify the effect of MT supplementation.

\section{Methods}

The present systematic review and meta-analysis was conducted based on the PRISMA guidelines. There was no completing interest.

Search strategy

PubMed, Embase, Google Scholar and the Cochrane Library were searched from the incipiency until April 2020. The keywords used to search for relevant literature were as follows: one about assisted reproductive technologies (assisted reproductive technology/ in vitro fertilization/ intracytoplasmic sperm injection/ ART/ IVF/ ICSI), one including terms on melatonin/ melatonin supplementation / MT, and the last one including outcome of IVF/ICSI (live birth, clinical pregnancy, fertilization). Merging the subsets with "AND" has produced more literatures. Papers published in non-English were excluded. Two reviewers reviewed the eligibility of included papers independently and the third reviewer was asked to review the paper when the discrepancy was happened.

\section{Study selection and data extraction}

Two authors reviewed title and abstract independently. Studies, which evaluated MT supplementation in women undergoing ART treatment, were included and analyzed. The main outcome index was the LBR, CPR, Number of oocyte/MII, Number of top embryo, fertilization rate (FR) and/or miscarriage rate (MR).

In this study, the data of each eligible study included were extracted and recorded in a $2 \times 2$ table. For incomplete data, we contacted the author or calculated from the existing data. In addition, we also recorded the type of study, interventions, sample size, inclusion and exclusion criteria, usage of MT, and all the outcomes reported in each study. Two authors completed the quality assessment, and the third author resolved any discrepancy.

\section{Data analysis}

RevMan 5.3 (Cochrane Collaboration, Oxford, UK) was applied for statistical analysis. The Risk Ratio (RR) with the $2 \times 2$ tables was used for binary outcomes, and mean difference (MD) for continuous outcomes. Forest plots were used to assess the heterogeneity of the included studies graphically and the $R^{2}$ value was used to quantitatively assess the heterogeneity between studies. The precision of the estimates were evaluated by $95 \%$ $\mathrm{Cl}$ via fixed or random effect model. Because of the low power of $X^{2}$ test for heterogeneity in of a meta-analysis, the statistical significant was considered when the $P$ value $<0.1$ rather than $<0.05$, especially in the studies with small sample size.

\section{Results}

\section{Studies selection and characteristics}

This search strategy produced 160 citations. After reviewing the title and/or abstract, 103 irrelevant studies were excluded. Of the remaining 57 papers, 44 studies were excluded, as outcomes of IVF/ICSI were not reported or 
irrelevant to our subject. Two studies were system review and meta-analysis. The study flow diagram is shown as Supplemental Table 1.

The characteristics of the included studies are showed as in Table 1. 11 prospective studies were included in our meta-analysis. In these studies, there were 10 studies for clinical pregnancy, 3 studies for live birth rate, 6 studies for miscarriage rate, 7 studies for fertilization rate, 9 (8) studies for oocyte (MII) number, and 6 studies for top embryo. The control group used placebo or no-treatment in 7 studies, and used standard treatment with myoinositol + folic acid (MI+ FA) in 3 studies. One study did not report the treatment in control group. Among 11 studies, 1 study included infertile women with disturbed sleep, 2 studies included women with PCOS, 3 studies with normal ovarian function, 1 study with DOR, 3 studies with previous low fertilization rate or poor quality embryo, and 1 study did not reported the characteristic of participant.

\section{Meta-analysis}

Ten, three, six seven, nine, eight, six studies were obtained in our meta-analysis to assess the effect of MT supplementation on CPR, LBR, MR, FR, Number of oocyte, Number of MII, Number of top embryo after ART treatment, respectively.

When evaluated the effects of MT supplementation on the CPR, 10 studies were analyzed including 593 cycles with MT supplementation and 516 cycles without MT. The CPR was significantly increased in women with MT supplementation compared with control group. There was no heterogeneity across studies with $P=0.96\left(I^{2}=0 \%\right)$. The fixed effects model was implied and the combined PR was 1.24 (95\% Cl, 1.04, 1.47; P=0.02) (Fig. 1).

Subsequently, we presented stratified results by the interventions. The MT supplementation with or without MI+FA did not bring beneficial effect on CPR with combined RR $1.22(95 \% \mathrm{Cl}, 0.96,1.54 ; \mathrm{P}=0.10)$ compared with treatment with Ml+FA and $1.26(95 \% \mathrm{Cl}, 0.97,1.62 ; \mathrm{P}=0.08)$ compared with treatment with placebo/none. Included studies were subgrouped into 4 types by the characteristic of women: women with PCOS (2 studies), Normal ovarian function (3 studies), with previous poor oocyte quality of low fertilization rate (3 studies), and DOR (1 study). The pooled RRs in women with PCOS, Normal ovarian function, and with previous poor oocyte quality/ low fertilization rate, were 1.18 (95\% Cl, 0.92, 1.52; $\mathrm{P}=0.18), 1.15$ (95\% Cl, 0.87, 1.53; $\mathrm{P}=0.32), 1.71$ (95\% $\mathrm{Cl}, 0.95,3.07 ; \mathrm{P}=0.07)$, respectively, showed no significant difference with control group.

Three studies were included to evaluate the effects of MT supplementation on the LBR, including 190 cycles with MT supplementation and 101 cycles in control group. The LBR was similar between groups. The statistical heterogeneity was good with $\mathrm{P}=0.80\left(\mathrm{I}^{2}=0 \%\right)$. The combined $\mathrm{RR}$ was $1.23(95 \% \mathrm{Cl}, 0.85,1.80 ; \mathrm{P}=0.27)($ Fig. 2$)$.

Of 11 studies, six studies evaluated the effect of MT supplementation on MR. These results indicated that there was no beneficial effect in patients with MT supplementation. The pooled RR for MR was $0.96(95 \% \mathrm{Cl}, 0.50$, 1.82; $P=0.89)$ with fixed effects model. There was no heterogeneity of the studies $\left(I^{2}=0 \%, P=0.91\right)$. (Sup Fig. 1)

As for the FR, three studies evaluated FR as mean \pm SD, and the pooled MD was 0.13 (95\% Cl, 0.01, 0.24; $\mathrm{P}=0.03)$, with moderate statistical heterogeneity $\left(\mathrm{I}^{2}=73 \%, \mathrm{P}=0.01\right)$. In addition, four studies assessed FR as cases (events/ total), and the pooled RR was $1.10(95 \% \mathrm{Cl}, 1.03,1.17 ; \mathrm{P}=0.007)$ with moderate statistical heterogeneity $\left(I^{2}=49 \%, P=0.12\right)$. (Sup Fig. 2) 
We also evaluated the effect of MT supplementation on the Number of oocyte (Sup Fig. 3), MII (Sup Fig. 4) and top-quality embryo (Sup Fig. 5). The results showed MT supplementation increased number of MII (MD 1.39; $95 \% \mathrm{Cl}, 0.74,2.04 ; \mathrm{P}<0.0001$ ) and top-quality embryo (MD 0.56; 95\% Cl, 0.24, 0.88; $\mathrm{P}=0.0005$ ) compared with control group. However, there was no difference in number of oocyte (MD 0.58; 95\% Cl, $-0.12,1.27 ; P=0.10$ ). Regardless of whether the intervention is with or without MI+FA, the supplementation of MT can significantly increase the number of MII (MI+MT+FA vs. MI+FA: MD 0.91; 95\% CI 0.40, 1.41; P=0.004; MT vs. placebo/none: MD 2.06, 95\% Cl 0.73, 3.39; P=0.002), and top-quality embryo (Ml+MT+FA vs. MI+FA: MD 0.70; 95\% Cl 0.24, 1.16; $\mathrm{P}=0.003$; $\mathrm{MT}$ vs. placebo/none: MD 0.33, 95\% Cl 0.11, 0.54; $\mathrm{P}=0.003$ ). However, MT supplementation only increased the No. of MII in women with PCOS (MD 0.97; 95\% Cl 0.22, 1.73; $\mathrm{P}=0.0004)$, and not in women with normal ovarian function (MD 1.49; $95 \% \mathrm{Cl}-0.33,3.31$; $\mathrm{P}=0.11$ ) compared with control group.

These studies included were all have medium to high score via the Newcastle-Ottawa Quality Assessment Scale (not shown). There was no publication bias as a symmetrical shape was seen in the funnel plot in the evaluation the effect of MT supplementation on CPR, LBR, MR, Number of oocyte, MII, and top-quality embryo. However, the studies showed modest publication bias when assess the effect of MT supplementation on FR. (Sup Fig. 6-12)

\section{Discussion}

So far, two systematic reviews have evaluated whether MT supplementation improve the outcomes of IVF/ICSI treatments, but drawn different conclusions. As far as we know, this study is the one with the largest sample size of 1303 IVF/ICSI cycles.

In the present systematic review, 10, 3, 6, 7, 9, 8, 6 studies were included assessing the effect of MT supplementation on the CPR, LBR, MR, FR, Number of oocyte, MII, Top-quality embryo after IVF/ICSI, respectively. Our results indicated that MT supplementation could improve the CPR significantly (combined RR $1.24 ; 95 \% \mathrm{Cl}$ 1.04, 1.47; P=0.02), FR (3 studies: MD 0.13; 95\% Cl, 0.01, 0.24; P=0.03; 4 studies: RR 1.10; 95\% Cl, 1.03, 1.17; $\mathrm{P}=0.007$ ), Number of MII (MD 1.39; $95 \% \mathrm{Cl}, 0.74,2.04 ; \mathrm{P}<0.0001)$, and top-embryo (MD 0.56; $95 \% \mathrm{Cl}, 0.24,0.88$; $\mathrm{P}=0.0005)$; however, we did not found significant differences in LBR, MR and Number of oocyte between groups.

However, when the studies were stratifies by the interventions or by characteristic of participants, there were almost completely different results: 1) No difference in CPR whatever the interventions are and whatever the participants are; 2) significantly increased Number of MII and top embryo in regardless of the interventions; increased No. of MII in women with PCOS, and not in with normal ovarian function.

The conclusion was not completely agreement with the systematic reviews by Seko et al. (10) and Hu et al. (11). The former, only included 5 studies, did not report LBR, and did not show beneficial effect on the CPR, Number of oocyte. The latter, which included one IUI study (3) and one study with in-vitro application of MT (12), suggested that the MT supplementation increase the CPR, Number of oocyte, MII, and good quality embryo but not live birth rate in ART cycles. But, this study included incomplete data, and the calculation method of miscarriage rate is not suitable.

In the ART process, controlled ovarian stimulation (COS), in vitro incubation of oocyte/embryo and fertilization may leave the oocyte/embryo in a high ROS environment compared with the physiological condition. Furthermore, excessive ROS results in damage of telomeres (13), loss of cell membrane integrity and change in 
functional structures (14), leading to poor quality oocyte/embryo and unsatisfactory pregnancy outcome. In order to obtain a high-quality embryo, the oxidative stress from ROS must be limited.

Antioxidant treatment is one of the mechanisms to combat the oxidative stress. The effects of MT on female reproduction has been summarized by Tamura et al. (15). Several human and animal studies suggested that MT could be used to treat infertility (16-17). Many clinical trials have shown that MT supplementation has positive effect on the quality of oocytes and embryo, leading to improved outcomes of IVF (6-8). However, some other studies did not show such improved outcomes $(9,18)$.

Consistent with other studies, our pooled results showed MT have beneficial effect on oocyte quality and embryo quality, especially in women with PCOS. As we all known, one main future of PCOS is oocyte maturation disorder (19). There were some possible explanations to our results as follows: 1) MT receptors (MTR) are present in granulosa cells, oocytes and embryos (14, 20-21), which laid foundation for MT's function. 2) MT is amphiphilic, and it can easily pass through cell membranes (4). 3) Both MT and its metabolites are terminal stable antioxidant, will not act as an oxidant in any case $(22,23)$.

In addition to its powerful free-radical scavenging effect, MT has immunomodulatory effect, can promote the secretion of progesterone, and can inhibit the synthesis of prostaglandins, which may increase the risk of miscarriage and premature delivery (24). Animal study on rats have shown that MT can enhance the expression of MT receptor and p53 receptor, so MT can affect the morphology of the endometrium and increase embryo implantation (25). Theoretically, MT could be helpful for pregnancy establishment and pregnancy maintenance. In the case of spontaneous abortions that exclude chromosomal anomalities and/or uterine abnormalities, MT was reported to be a protective factor for spontaneous abortion (4). Regretly, our results did not showed significantly beneficial effect of MT supplementation on CPR, MR, and LBR.

We also analyzed the possible reasons: 1) MT has a fast metabolism and a short half-life, so in the IVF cycle, the blood concentration of a single daily dosing may not achieve effective antioxidant effects. 2) The initiating time and duration of MT treatment are also related to the effects of the treatment, which was variable between studies. 3) The successful pregnancy after IVF/ICSI was influenced by many factors, and the beneficial effect may be offset by other potential disadvantage.

A strength of systematic reviews lies in the comprehensive evaluation of the results of individual studies. The present systematic review indicated that MT supplementation has an effect on FR, quality of oocyte and embryo after IVF/ICSI. Our study enrolled the largest sample size, and firstly evaluated the fertilization. Besides, we tried our best to extract the completed data by contacting with authors or by calculating based on published data dealing with missing data.

Of course, this meta-analysis also has weakness, and the main one is the variable characteristics between the included studies: different participants, different interventions (MI+FA+MT, MT), and different dose and duration of MT, different inclusion/ exclusion criteria, and different outcomes. Besides, some possible confounding factors, such as smoker or non-smoker, number of previous failed IVF cycle, were not controlled.

\section{Conclusion}


The present systematic review suggests that MT supplementation have beneficial effect on the maturation of oocyte, fertilization and quality of embryo. Existing evidence did not suggest that MT supplementation has significant effect on the LBR, CPR, and MR. The MT supplementation may be recommended to women with PCOS, with previous poor oocyte quality/ low fertilization.

\section{Abbreviations}

ART: Assisted reproductive technology; IVF-ET: In vitro fertilization - embryo transfer; ICSI: Intracytoplasmic sperm injection; MT: Melatonin; RR: Risk Ratio; MD: Mean difference; CPR: Clinical pregnancy rate; LBR: Live birth rate; MR: Miscarriage rate; FR: Fertilization rate; Cl: Confidence interval; MI: Myoinositol; FA: Folic acid; DOR: Decreased ovarian reserve; PCOS: Polycystic ovary syndrome; ROS: Reactive oxygen species

\section{Declarations}

\section{Acknowledgement}

There was no acknowledgement

\section{Funding}

No funding

\section{Availability of data and material}

All data is available in this paper.

\section{Authors' Contributions}

Jing Zhao participated in the design of the study and the acquisition of data, performed the statistical analysis, drafted the article and revised it critically. Yanping Li contributed to conception and design. Jie Hao conducted acquisition of data and analysis and interpretation of data. Bin Xu and Yonggang Wang participated in the interpretation of the data and the revision of the article. All authors read and approved the final manuscript.

\section{Ethics approval and consent to participate}

Not applicable

\section{Consent for publication}

No applicable

\section{Competing interests}

The authors declare that they have no conflict of interest

\section{References}


1. Calhaz-Jorge C, De Geyter C, Kupka MS, De Mouzon J, Erb K, Mocanu E, et al. Assisted reproductive technology in Europe, 2012: Results generated from European registers by ESHRE. Hum Reprod 2016;31(8):1638-52.

2. Malchau SS, Henningsen AA, Forman J, Loft A, Nyboe Andersen A, Pinborg A. Cumulative live birth rate prognosis based on the number of aspirated oocytes in previous ART cycles. Hum Reprod 2019;34(1):17180.

3. Mokhtari F, Asbagh FA, Azmoodeh O, Bakhtiyari M, Almasi-Hashiani A. Effects of melatonin administration on chemical pregnancy rates of polycystic ovary syndrome patients undergoing intrauterine insemination: $A$ randomized clinical trial. Int J Fertil Steril 2019;13(3):225-9.

4. Tamura H, Jozaki M, Tanabe M, Shirafuta Y, Mihara Y, Shinagawa M, et al. Importance of Melatonin in Assisted Reproductive Technology and Ovarian Aging. Int J Mol Sci 2020;21(3).

5. Fernández A, Ordõñez R, Reiter RJ, González-Gallego J, Mauriz JL. Melatonin and endoplasmic reticulum stress: Relation to autophagy and apoptosis. J Pineal Res 2015;59(3):292-307.

6. Batiioğlu AS, Şahin U, Grlek B, Öztrk N, Ünsal E. The effıcacy of melatonin administration on oocyte quality. Gynecol Endocrinol 2012;28(2):91-3.

7. Espino J, Macedo M, Lozano G, Ortiz Á, Rodríguez C, Rodríguez AB, et al. Impact of melatonin supplementation in women with unexplained infertility undergoing fertility treatment. Antioxidants 2019;8(9).

8. Jahromi BN, Sadeghi S, Alipour S, Parsanezhad ME, Alamdarloo SM. Effect of melatonin on the outcome of assisted reproductive technique cycles in women with diminished ovarian reserve: A double-blinded randomized clinical trial. Iran J Med Sci 2017;42(1):73-8.

9. Rizzo P, Raffone E, Benedetto V. Effect of the treatment with myo-inositol plus folic acid plus melatonin in comparison with a treatment with myo-inositol plus folic acid on oocyte quality and. Eur Rev Med Pharmacol [Internet] 2010;14:555-61. Available from: https://www.europeanreview.org/wp/wpcontent/uploads/780.pdf\%0Ahttp://www.haldanepharma.com/images/products/16032807223203_Myoino Oocyte quality.pdf

10. Seko LMD, Moroni RM, Leitao VMS, Teixeira DM, Nastri CO, Martins WP. Melatonin supplementation during controlled ovarian stimulation for women undergoing assisted reproductive technology: Systematic review and meta-analysis of randomized controlled trials. Fertil Steril [Internet] 2014;101(1):154-161.e4. Available from: http://dx.doi.org/10.1016/j.fertnstert.2013.09.036

11. Hu K-L, Ye X, Wang S, Zhang D. Melatonin Application in Assisted Reproductive Technology: A Systematic Review and Meta-Analysis of Randomized Trials. Front Endocrinol (Lausanne) 2020;11(March).

12. Kim MK, Park EA, Kim HJ, Choi WY, Cho JH, Lee WS, et al. Does supplementation of in-vitro culture medium with melatonin improve IVF outcome in PCOS. Reprod Biomed Online [Internet] 2013;26(1):22-9. Available from: http://dx.doi.org/10.1016/j.rbmo.2012.10.007

13. Richter T, Zglinicki T von. A continuous correlation between oxidative stress and telomere shortening in fibroblasts. Exp Gerontol 2007;42(11):1039-42.

14. Hiroshi Tamura, Akihisa Takasaki, Toshiaki Taketani, Manabu Tanabe, Fumie Kizuka, Lifa Lee, Isao Tamura, Ryo Maekawa, Hiromi Aasada YY and NS. The Role of melatonin as an antioxidant in the follicle. J Ovarian Res 2012;5(MAY):1-9. 
15. Tamura H, Takasaki A, Taketani T, Tanabe M, Lee L, Tamura I, et al. Melatonin and female reproduction. J Obstet Gynaecol Res 2014;40(1):1-11.

16. Ishizuka B, Kuribayashi Y, Murai K, Amemiya A, Itoh MT. The effect of melatonin on in vitro fertilization and embryo development in mice. J Pineal Res 2000;28(1):48-51.

17. Berlinguer F, Leoni GG, Succu S, Spezzigu A, Madeddu M, Satta V, et al. Exogenous melatonin positively influences follicular dynamics, oocyte developmental competence and blastocyst output in a goat model. J Pineal Res 2009;46(4):383-91.

18. Eryilmaz OG, Devran A, Sarikaya E, Aksakal FN, Mollamahmutoğlu L, Cicek N. Melatonin improves the oocyte and the embryo in IVF patients with sleep disturbances, but does not improve the sleeping problems. J Assist Reprod Genet 2011;28(9):815-20.

19. Mojaverrostami S, Asghari N, Sc M, Khamisabadi M, Khoei HH, Abbas A. The role of melatonin in polycystic ovary syndrome: A review Production and Hosting by Knowledge E. Int J Reprod Biomed 2019;17(12):86582.

20. Woo MMM, Tai CJ, Kang SK, Nathwani PS, Pang SF, Leung PCK. Direct action of melatonin in human granulosa-luteal cells. J Clin Endocrinol Metab 2001;86(10):4789-97.

21. Pang $Y$, Zhao S, Sun $Y$, Jiang $X, \mathrm{Hao} H$, Du W, et al. Protective effects of melatonin on the in vitro developmental competence of bovine oocytes. Anim Sci J 2018;89(4):648-60.

22. Galano A, Tan DX, Reiter RJ. On the free radical scavenging activities of melatonin's metabolites, AFMK and AMK. J Pineal Res 2013;54(3):245-57.

23. García JJ, Lõpez-Pingarrõn L, Almeida-Souza P, Tres A, Escudero P, García-Gil FA, et al. Protective effects of melatonin in reducing oxidative stress and in preserving the fluidity of biological membranes: A review. J Pineal Res 2014;56(3):225-37.

24. Carlomagno G, Minini M, Tilotta M, Unfer V. From implantation to birth: Insight into molecular melatonin functions. Int J Mol Sci 2018;19(9).

25. Lu Zhang, Zhenzhen Zhang, Feng Wang, Xiuzhi Tian PJ and GL. Effects of melatonin administration on embryo implantation and offspring growth in mice under different schedules of photoperiodic exposure. Reprod Biol Endocrinol 2017;15(1):1-9.

\section{Tables}

Table 1-2 are available in the Supplementary Files

\section{Figures}



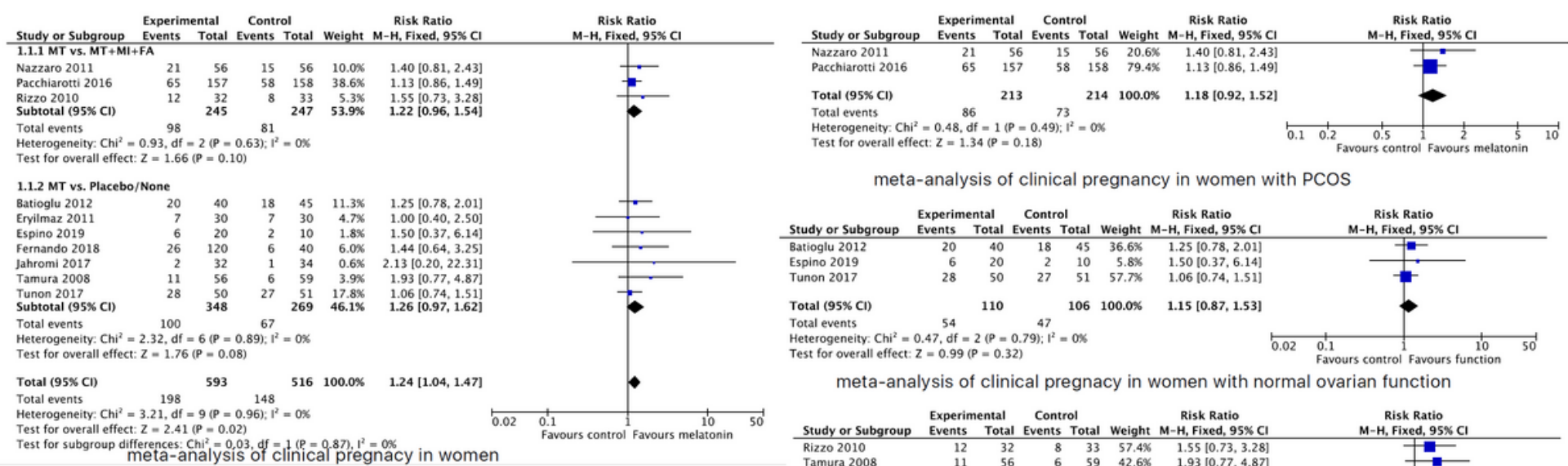

meta-analysis of clinical pregnancy in women with PCOS

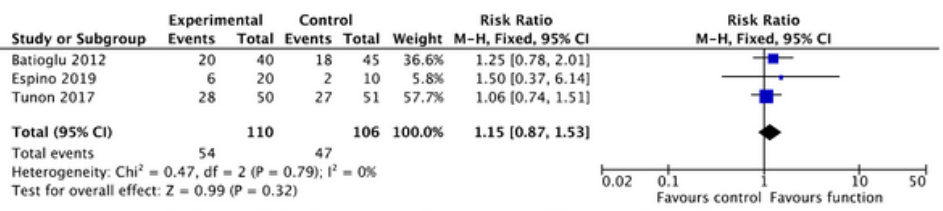

meta-analysis of clinical pregnacy in women with normal ovarian function

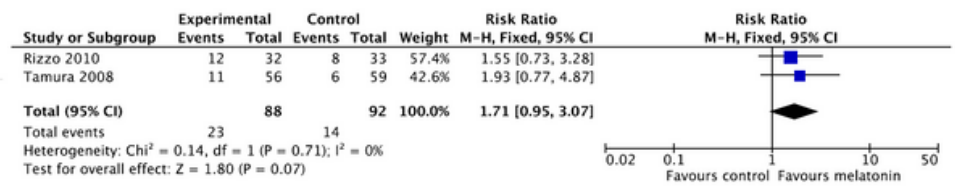

meta-analysis of clinical pregnacy in women with previous poor oocyte/ embryo quality or low fertilization rate

\section{Figure 1}

Forest plot showing the results of meta-analysis of studies comparing the effect of MT supplementation on clinical pregnancy rate after IVF/ICSI.

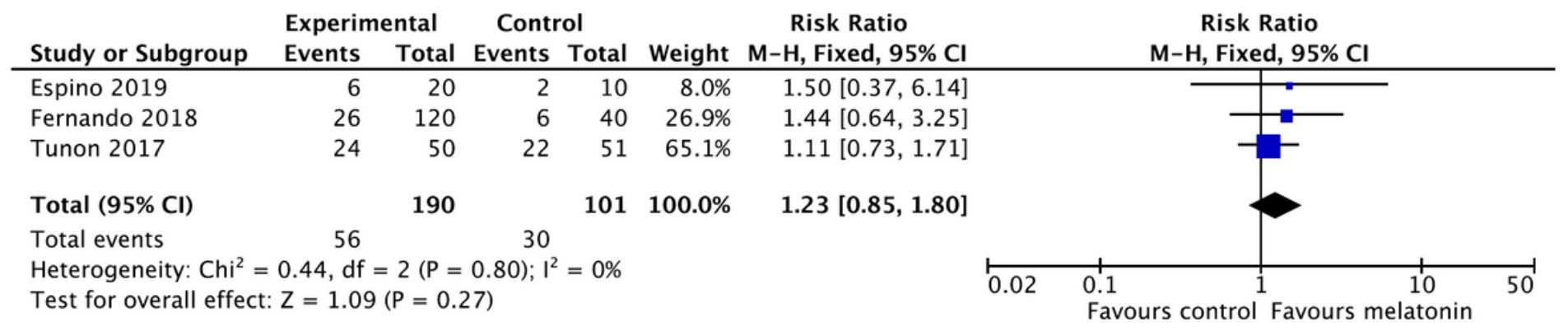

\section{meta-analysis of studies reporting live birth rate}

\section{Figure 2}

Forest plot showing the results of meta-analysis of studies comparing the effect of MT supplementation on live birth rate after IVF/ICSI.

\section{Supplementary Files}

This is a list of supplementary files associated with this preprint. Click to download.

- SupFigure1MRForestplot.pdf

- SUPFigure2FRForestplot.pdf

- SUPFigure3NumberofoocyteForestplot.pdf

- SUPFigure4No.ofMIIForestplot.pdf

- SUPFigure5No.ofTopembryoForestplot.pdf 
- SupFig6CPRFunnelplot.pdf

- SupFig7LBRFunnelplot.pdf

- SupFig8MRFunnelplot.pdf

- SupFig9FRFunnelplot.pdf

- SupFig10No.oocyteFunnelplot.pdf

- SupFig11No.ofMIIFunnelplot.pdf

- SupFig12No.oftopembryoFunnelplot.pdf

- SUPTable1flowchart.doc

- Table1flowchart.doc

- Table2Characteristicofstudies.docx 